\title{
Domination of sample maxima and related extremal dependence measures
}

https://doi.org/10.1515/demo-2018-0005

Received October 31, 2017; accepted April 9, 2018

Abstract: For a given $d$-dimensional distribution function (df) $H$ we introduce the class of dependence measures $\mu(H, Q)=-\mathbb{E}\left\{\ln H\left(Z_{1}, \ldots, Z_{d}\right)\right\}$, where the random vector $\left(Z_{1}, \ldots, Z_{d}\right)$ has $\mathrm{df} Q$ which has the same marginal dfs as $H$. If both $H$ and $Q$ are max-stable dfs, we show that for a $\mathrm{df} F$ in the max-domain of attraction of $H$, this dependence measure explains the extremal dependence exhibited by $F$. Moreover, we prove that $\mu(H, Q)$ is the limit of the probability that the maxima of a random sample from $F$ is marginally dominated by some random vector with $\mathrm{df}$ in the max-domain of attraction of $Q$. We show a similar result for the complete domination of the sample maxima which leads to another measure of dependence denoted by $\lambda(Q, H)$. In the literature $\lambda(H, H)$, with $H$ a max-stable df, has been studied in the context of records, multiple maxima, concomitants of order statistics and concurrence probabilities. It turns out that both $\mu(H, Q)$ and $\lambda(Q, H)$ are closely related. If $H$ is max-stable we derive useful representations for both $\mu(H, Q)$ and $\lambda(Q, H)$. Our applications include equivalent conditions for $H$ to be a product $\mathrm{df}$ and $F$ to have asymptotically independent components.

Keywords: Max-stable distributions, domination of sample maxima, extremal dependence, inf-argmax formula, de Haan representation, records, multiple maxima, concomitants of order statistics, concurrent probabilities

MSC: $60 \mathrm{G} 15,60 \mathrm{G} 70$

\section{Introduction}

Let $H$ be a $d$-dimensional distribution function (df) with unit Fréchet marginal dfs $\Phi(x)=e^{-1 / x}, x>0$. We shall assume in the sequel that $H$ is a max-stable df, which in our setup is equivalent to the homogeneity property

$$
H^{t}\left(x_{1}, \ldots, x_{d}\right)=H\left(t x_{1}, \ldots, t x_{d}\right),
$$

for any $t>0, x_{i} \in(0, \infty), 1 \leq i \leq d$, see for instance $[2,9,25]$. The class of max-stable dfs is very large with the two extreme instances

$$
H_{0}\left(x_{1}, \ldots, x_{d}\right)=\prod_{i=1}^{d} \Phi\left(x_{i}\right) \quad \text { and } \quad H_{\infty}\left(x_{1}, \ldots, x_{d}\right)=\min _{1 \leq i \leq d} \Phi\left(x_{i}\right),
$$

the product df $H_{0}$ and the upper df $H_{\infty}$, respectively. Hereafter $\bar{G}=1-G$ stands for the survival function of some univariate $\mathrm{df} G$. It follows easily by the lower Fréchet -Hoeffding bound that

$$
\left(H\left(n x_{1}, \ldots, n x_{d}\right)\right)^{n} \geq\left(\max \left(0,1-\sum_{i=1}^{d} \bar{\Phi}\left(n x_{i}\right)\right)\right)^{n} \geq e^{\liminf n_{n \rightarrow \infty} n \ln \left(1-\sum_{i=1}^{d} \bar{\Phi}\left(n x_{i}\right)\right)}
$$

\footnotetext{
^Corresponding Author: Enkelejd Hashorva: Department of Actuarial Science University of Lausanne, UNIL-Dorigny, 1015
} Lausanne, Switzerland, E-mail: Enkelejd.Hashorva@unil.ch 


$$
=H_{0}\left(x_{1}, \ldots, x_{d}\right), \quad x_{i} \in(0, \infty), \quad i \leq d .
$$

Indeed, (1.2) is well-known and follows for instance using the Pickands representation of $H$, see [9, Eq. (4.3.1)] or the inf-argmax formula as shown in Section 4. Consequently, any max-stable df $H$ lies between $H_{0}$ and $H_{\infty}$, i.e.,

$$
H_{0}\left(x_{1}, \ldots, x_{d}\right) \leq H\left(x_{1}, \ldots, x_{d}\right) \leq H_{\infty}\left(x_{1}, \ldots, x_{d}\right), \quad x_{i} \in(0, \infty), 1 \leq i \leq d
$$

From multivariate extreme value theory, see e.g. [2, 4, 9, 25], we know that $d$-dimensional max-stable dfs $H$ are limiting dfs of the component-wise maxima of $d$-dimensional independent and identically distributed (iid) random vectors with some $\mathrm{df} F$. In that case, $F$ is said to be in the max-domain of attraction (MDA) of $H$ (abbreviated $F \in M D A(H)$ ). For simplicity we shall assume throughout in the following that $F$ is a df on $[0, \infty)^{d}$ with marginal dfs $F_{i} \in M D A(\Phi), i \leq d$ that have norming constants $a_{n}=n, n \in \mathbb{N}$, and thus we have

$$
\lim _{n \rightarrow \infty} F_{i}^{n}(n x)=\Phi(x), \quad x \in \mathbb{R}
$$

for all $i \leq d$, where we set $\Phi(x)=0$ if $x \leq 0$. Consequently, $F$ is in the MDA of some max-stable df $H$ if further

$$
\lim _{n \rightarrow \infty} \sup _{x_{i} \in \mathbb{R}, 1 \leq i \leq d}\left|F^{n}\left(n x_{1}, \ldots, n x_{d}\right)-H\left(x_{1}, \ldots, x_{d}\right)\right|=0
$$

In the special case that $F$ has asymptotically independent marginal dfs, meaning that for $\left(X_{1}, \ldots, X_{d}\right)$ with df $F$

$$
\lim _{n \rightarrow \infty} n \mathbb{P}\left\{X_{i}>n x_{i}, X_{j}>n x_{j}\right\}=0, \quad x_{i}, x_{j} \in(0, \infty), \quad \forall i=j \leq d
$$

then $F \in M D A\left(H_{0}\right)$ if simply $F_{i} \in M D A(\Phi), i \leq d$.

In various applications it is important to be able to determine if some max-stable $\mathrm{df} H$ resulting from the approximation in (1.5) is equal to $H_{0}$, which in the light of multivariate extreme value theory means that the component-wise maxima $\boldsymbol{M}_{n}:=\left(\max _{1 \leq i \leq n} X_{i 1}, \ldots, \max _{1 \leq i \leq n} X_{i d}\right), n \geq 1$ of a $d$-dimensional random sample $\left(X_{i 1}, \ldots, X_{i d}\right), i=1, \ldots, n$ of size $n$ from $F$ has asymptotically independent components.

The strength of dependence of the components of $\boldsymbol{M}_{n}$, or in other words the extremal dependence manifested in $F$, in view of the approximation (1.5) can be measured by calculating some appropriate dependence measure for $H$ (when the limiting df $H$ is known).

For any random vector $\boldsymbol{Z}=\left(Z_{1}, \ldots, Z_{d}\right)$ with $\mathrm{df} Q$ which has the same marginal dfs as $H$ we introduce a class of dependence measure for $H$ indexed by $Q$ given by

$$
\mu(H, Q)=-\mathbb{E}\left\{\ln H\left(Z_{1}, \ldots, Z_{d}\right)\right\} .
$$

In view of (1.3), since $-\ln H_{i}\left(Z_{i}\right)$ is a unit exponential random variable, we have

$$
1=\max _{1 \leq i \leq d} \mathbb{E}\left\{-\ln H_{i}\left(Z_{i}\right)\right\} \leq-\mathbb{E}\left\{\ln \min _{1 \leq i \leq d} H_{i}\left(Z_{i}\right)\right\} \leq \mu(H, Q) \leq-\mathbb{E}\left\{\ln \prod_{i=1}^{d} H_{i}\left(Z_{i}\right)\right\}=d,
$$

and, in particular:

$$
\mu\left(H_{0}, Q\right)=d, \quad \mu\left(H_{\infty}, H_{\infty}\right)=1
$$

Clearly, $\mu(H, Q)$ can be defined for any df $H$ and it does not depend on the choice of the marginal dfs of $H$. In this contribution we shall show that $\mu(H, Q)$ is particularly interesting for $H$ being max-stable.

Next, consider the case that both $H$ and $Q$ are max-stable. It follows that (see Theorem 2.3) for $F$ satisfying (1.5) and $G \in M D A(Q)$

$$
\mu(Q, H)=\lim _{n \rightarrow \infty} \mu_{n}\left(G, F^{n}\right), \quad \mu_{n}\left(G, F^{n}\right)=n \int_{\mathbb{R}^{d}}\left[1-G\left(x_{1}, \ldots, x_{d}\right)\right] d F^{n}\left(x_{1}, \ldots, x_{d}\right),
$$


provided that both $F$ and $G$ are continuous. In view of (1.10), we see that $\mu(H, Q)$ relates to $F$ under (1.5). Let in the following $\boldsymbol{W}$ denote a random vector with $\mathrm{df} G$ being independent of $\boldsymbol{M}_{n}$. We say that $\boldsymbol{W}$ marginally dominates $\boldsymbol{M}_{n}$, if there exists some $i \leq d$ such that $W_{i}>M_{n i}$. Consequently, assuming further that $\boldsymbol{W}$ is independent of $\boldsymbol{M}_{n}$ we have

$$
\frac{\mu_{n}\left(G, F^{n}\right)}{n}=\mathbb{P}\left\{\boldsymbol{W} \text { marginally dominates } \boldsymbol{M}_{n}\right\}=: \underline{\pi}_{n} .
$$

Re-writing (1.10) we have $\lim _{n \rightarrow \infty} n \underline{\pi}_{n}=\mu(H, Q)$ and thus $\mu(H, Q)$ appears naturally in the context of marginal dominance of sample maxima.

Our motivation for introducing $\mu(H, Q)$ comes from results and ideas of A. Gnedin, see $[10,12,13]$ where multiple maxima of random samples is investigated. In the turn, the probability of observing a multiple maximum is closely related to the complete domination of sample maxima as we shall explain below.

We say that $\boldsymbol{W}$ completely dominates $\boldsymbol{M}_{n}$ if $W_{i}>M_{n i}$ for any $i \leq d$. Assuming that $F$ and $G$ are continuous, we have

$$
\lambda_{n}\left(F^{n}, G\right):=n \int_{\mathbb{R}^{d}} F^{n}\left(x_{1}, \ldots, x_{d}\right) d G\left(x_{1}, \ldots, x_{d}\right)=n \mathbb{P}\left\{\boldsymbol{W} \text { completely dominates } \boldsymbol{M}_{n}\right\}=: n \bar{\pi}_{n} .
$$

If further $F \in M D A(H), G \in M D A(Q)$ we show in Theorem 2.3 below that

$$
\lim _{n \rightarrow \infty} \lambda_{n}\left(G^{n}, F\right)=\lambda(Q, H)=\int_{(0, \infty)^{d}} Q\left(x_{1}, \ldots, x_{d}\right) d v\left(x_{1}, \ldots, x_{d}\right),
$$

where $v$ denotes the exponent measure of $H$ defined on $E=[0, \infty]^{d} \backslash(0, \ldots, 0)$, see $[9,25]$ for more details on the exponent measure. Note in passing that by symmetry $\lim _{n \rightarrow \infty} \lambda_{n}\left(F^{n}, G\right)=\lambda(H, Q)$ follows.

Our notation and definitions of $\bar{\pi}_{n}$ and $\underline{\pi}_{n}$ agree with those in [7] for the particular case that $F=G$. Therein the complete and simple records are discussed. If $F$ is continuous and $F=G$ we have that $(n+1) \bar{\pi}_{n}$ equals

$$
\mathbb{P}\left\{\max _{1 \leq i \leq n+1} X_{i j}=X_{1 j}, j=1, \ldots, d\right\},
$$

which is the probability of observing a multiple maximum, see [8, 11-13, 20, 21]. There are only few contributions that discuss the asymptotics of $\lambda_{n}\left(G^{n}, F\right)$ for $F /=G$, see $[16,17,19]$.

Since the exponent measure can be defined also for max-id df $H$, i.e., if $H^{t}$ is a df for any $t>0$, then as above $\lambda(Q, H)$ can also be defined for any such df $H$ and any given $d$-dimensional $\mathrm{df} Q$. We shall show that $\mu(H, Q)$ and $\lambda(Q, H)$ are closely related. In particular, for $d=2$ we have $\mu(H, Q)=2-\lambda(Q, H)$, provided that $H$ is a max-id df. In particular, we show how to define $\lambda(Q, H)$ for any $H$ and $Q$.

For $H$ being a max-id df we also show how to calculate $\mu(H, Q)$ and $\lambda(Q, H)$ by a limiting procedure, which relates to domination of $d$-dimensional random vectors, see Theorem 2.1 below.

It turns out that both dependence measures $\mu(H, Q)$ and $\lambda(Q, H)$ are very tractable if $H$ is max-stable (note that such $H$ is also max-id df). In particular, we show that $\mu(H, Q)$ is the extremal coefficient of some $d$ dimensional max-stable $\mathrm{df} H^{\star}$, i.e., $\mu(H, Q)=-\ln H^{\star}(1, \ldots, 1)$. Moreover, we derive in Theorem 2.5 tractable expressions for $\mu(H, Q)$ and $\lambda(Q, H)$, which are useful for simulations of these dependence measures if the de Haan spectral representation of $H$ is known.

It is of particular interest for multivariate extreme value theory to derive tractable criteria that identify if a max-stable df $H$ is equal to $H_{0}$. In our first application we show several equivalent conditions to $H=H_{0}$.

In view of (1.10) and (1.11) we see that both measures of extremal dependence $\mu(H, Q)$ and $\lambda(Q, H)$ capture the extremal properties of $F \in M D A(H)$. Motivated by the relation between $\mu(H, Q)$ and $\lambda(Q, H)$ we derive in our second application several conditions equivalent to (1.6).

Both $\mu(H, Q)$ and $\lambda(Q, H)$ can be defined for any $d$-dimensional df $H$ and $Q$. When $H$ is max-stable, these are dependence measures for $H$, since independent of the choice of $Q$, we can determine if $H=H_{0}$, see Proposition 3.1, statement ii). A simple choice for $Q$ is taking $Q=H$. Alternatively, one can take $Q=H_{0}$ or $Q=H_{\infty}$. Independent of the choice of $Q$ we show in Proposition 3.1 that $\mu(H, Q)=2$ is equivalent to $H=H_{0}$. In particular, this result shows that $\mu(H, Q)$ is a measure of dependence of $H$ (and not for $Q$ ). 
A summary of the remainder of the paper follows. In Section 2, we derive the basic properties of both measures of $\mu(H, Q)$ and $\lambda(Q, H)$ if $H$ is a max-id df. More tractable formulas are then derived for $H$ being a max-stable df. Section 3 is dedicated to applications. We present some auxiliary results in Section 4 followed by the proofs of the main results in Section 5 .

\section{Main Results}

In the following $H$ and $Q$ are $d$-dimensional dfs with unit Fréchet marginals $\mathrm{dfs}$ and $\boldsymbol{Z}$ is a random vector with df $Q$. The second dependence measure $\lambda(Q, H)$ defined in (1.11) is determined in terms of the exponent measure $v$ of $H$, under the max-stability assumption on $H$.

A larger class of multivariate dfs is that of max-id dfs. Recall that $H$ is max-id, if $H^{t}$ is a df for an $t>0$. For such dfs the corresponding exponent measure can be constructed, see for example [25], and therefore we can define $\lambda(Q, H)$ as in the Introduction for any $H$ a max-id $\mathrm{df}$ and any given $\mathrm{df} Q$. Note that any max-stable $\mathrm{df}$ is a max-id df, therefore in the following we shall consider first the general case that $H$ is a max-id df, and then focus on the more tractable case that $H$ is a max-stable df.

\subsection{Max-id df $H$}

Our analysis shows that $\mu(H, Q)$ and $\lambda(Q, H)$ are closely related. Specifically, if $d=2$, then $\mu(H, Q)=2$ $\lambda(Q, H)$, provided that $H$ is a max-id df. Such a relationship does not hold for the case $d>2$. However as we show below it is possible to calculate $\mu(H, Q)$ if we know $\lambda\left(Q_{K}, H_{K}\right)$ for any non-empty index set $K \subset$ $\{1, \ldots, d\}$. A similar result is shown for $\lambda(Q, H)$. In our notation $Q_{K}$ denotes the marginal df of $Q$ with respect to $K$ and $|K|$ stands for the number of the elements of the index set $K$. Below $\mu_{n}$ and $\lambda_{n}$ are as defined in the Introduction.

Theorem 2.1. If $H$ is a max-id $d f$, then we have

$$
\mu(H, Q)=\lim _{n \rightarrow \infty} \mu_{n}\left(H^{1 / n}, Q\right), \quad \lambda(Q, H)=\lim _{n \rightarrow \infty} \lambda_{n}\left(Q, H^{1 / n}\right) .
$$

Moreover,

$$
\mu(H, Q)=d+\sum_{2 \leq i \leq d}(-1)^{i+1} \sum_{K \subset\{1, \ldots, d\},|K|=i} \lambda\left(Q_{K}, H_{K}\right)
$$

and

$$
\lambda(Q, H)=d+\sum_{2 \leq i \leq d}(-1)^{i+1} \sum_{K \subset\{1, \ldots, d\},|K|=i} \mu\left(H_{K}, Q_{K}\right)
$$

Remark 2.2. i) For $H$ a max-stable df and $Q=H$ the claim in (2.3) is shown in [8, Th. 2.2, Eq. (13)].

ii) A direct consequence of (2.3) is that we can define $\lambda(Q, H)$ even if $H$ is not a max-id df by simply using the definition of $\mu\left(H_{K}, Q_{K}\right)$.

iii) It is clear that $\mu(H, Q) \geq \mu\left(H_{K}, Q_{K}\right)$ for any non-empty index set $K \subset\{1, \ldots, d\}$. Note that (2.1) shows that exactly the opposite relation holds for $\lambda(Q, H)$ when $H$ is a max-id df, namely

$$
\lambda(Q, H) \leq \lambda\left(Q_{K}, H_{K}\right) .
$$

In fact, (2.3) shows that we can calculate both $\mu(H, Q)$ and $\lambda(Q, H)$ by a limit procedure if we assume that $H$ is a max-id df, see for more details (5.1). Although such a limit procedure shows how to interpret these dependence measures in terms of domination of random vectors, it does not give a precise relation with extremal properties of random samples. Therefore in the following we shall restrict our attention to the tractable case that $H$ is a max-stable $\mathrm{df}$. 


\subsection{Max-stable df $H$}

We show next the relation of $\mu(H, Q)$ and $\lambda(Q, H)$ with the marginal and complete domination of sample maxima mentioned in the Introduction. Recall that in our notation $\bar{F}_{i}, \bar{G}_{i}, i \leq d$ stand for the marginal survival functions of $F$ and $G$, respectively.

Theorem 2.3. If $H, Q$ are max-stable $d f s$ with unit Fréchet marginals and $F, G$ are two d-dimensional continuous $d f$ s such that $\lim _{x \rightarrow \infty} \bar{F}_{i}(x) / \bar{G}_{i}(x)=c_{i} \in(0, \infty)$ for $i \leq d$ and further $F \in M D A(H), G \in M D A(Q)$, then (1.10) and (1.11) hold.

Remark 2.4. The relation $\lim _{n \rightarrow \infty} \lambda_{n}\left(F^{n}, F\right)=\lambda(H, H)$ for $F \in M D A(H)$ is known from works of A. Gnedin, see for instance [12, 13]. Explicit formulas are given in [22] for $d=2$. See also the recent contributions [7, 8].

In view of [4] (recall $H$ has unit Fréchet marginal dfs) the assumption that $H$ is max-stable implies the following de Haan representation (see e.g. [6, 23])

$$
-\ln H\left(x_{1}, \ldots, x_{d}\right)=\mathbb{E}\left\{\max _{1 \leq i \leq d} \frac{Y_{i}}{x_{i}}\right\}, \quad\left(x_{1}, \ldots, x_{d}\right) \in(0, \infty)^{d},
$$

where $Y_{j}$ 's are non-negative with $\mathbb{E}\left\{Y_{i}\right\}=1,1 \leq i \leq d$. As shown in [18], see also [3, 24] we have the alternative formula

$$
-\ln H\left(x_{1}, \ldots, x_{d}\right)=\sum_{i=1}^{d} \frac{1}{x_{i}} \Psi_{i}\left(x_{1}, \ldots, x_{d}\right), \quad\left(x_{1}, \ldots, x_{d}\right) \in(0, \infty)^{d},
$$

where $\Psi_{i}$ 's are non-negative zero-homogeneous, i.e., $\Psi_{i}\left(c x_{1}, \ldots, c x_{d}\right)=\Psi_{i}\left(x_{1}, \ldots, x_{d}\right)$ for any $c>0, x_{i} \in(0, \infty), i \leq d$. Moreover, $\Psi_{i}$ 's are bounded by 1 , which immediately implies the validity of the lower bound in (1.2).

In the literature $-\ln H(1, \ldots, 1)$ is also referred to as the extremal coefficient of $H$, denoted by $\theta(H)$, see for example [8].

Our next result gives alternative formulas for $\mu(H, Q)$ and shows that it is the extremal coefficient of the maxstable df $H^{*}$ defined by

$$
-\ln H^{*}\left(x_{1}, \ldots, x_{d}\right)=\mathbb{E}\left\{\max _{1 \leq i \leq d} \frac{Y_{i}}{x_{i} Z_{i}}\right\}, \quad\left(x_{1}, \ldots, x_{d}\right) \in(0, \infty)^{d},
$$

with $\boldsymbol{Z}$ being independent of $\boldsymbol{Y}=\left(Y_{1}, \ldots, Y_{d}\right)$. Note that since

$$
\mathbb{E}\left\{Y_{i}\right\}=\mathbb{E}\left\{1 / Z_{i}\right\}=1, \quad i \leq d
$$

and $Y_{i} / Z_{i}$ 's are non-negative, then $H^{\star}$ has unit Fréchet marginal dfs and moreover also $\tilde{H}$ defined by

$$
-\ln \tilde{H}\left(x_{1}, \ldots, x_{d}\right)=\mathbb{E}\left\{\max _{1 \leq i \leq d} \frac{1}{x_{i} Z_{i}}\right\}, \quad\left(x_{1}, \ldots, x_{d}\right) \in(0, \infty)^{d},
$$

is a max-stable df with unit Fréchet marginal dfs.

Theorem 2.5. If $H$ is a max-stable $d f$ with unit Fréchet marginal $d f s$ and de Haan representation (2.6) with $\boldsymbol{Y}$ being independent of $\boldsymbol{Z}$ with $d f Q$ which has unit Fréchet marginal $d f$ s, then we have

$$
\mu(H, Q)=\mathbb{E}\left\{\max _{1 \leq i \leq d} \frac{Y_{i}}{Z_{i}}\right\}=\sum_{i=1}^{d} \mathbb{E}\left\{\frac{1}{Z_{i}} \Psi_{i}\left(Z_{1}, \ldots, Z_{d}\right)\right\}, \quad\left(x_{1}, \ldots, x_{d}\right) \in(0, \infty)^{d},
$$

and

$$
\lambda(Q, H)=\mathbb{E}\left\{\min _{1 \leq i \leq d} \frac{Y_{i}}{Z_{i}}\right\} .
$$


Moreover, with $H^{\star}$ defined in (2.6), we have

$$
\mu(H, Q)=\theta\left(H^{\star}\right) \geq \max (\theta(H), \theta(\tilde{H})) \geq 1,
$$

and

$$
\lambda(Q, H) \leq \min \left(\mathbb{E}\left\{\min _{1 \leq i \leq d} Y_{i}\right\}, \mathbb{E}\left\{\min _{1 \leq i \leq d} \frac{1}{Z_{i}}\right\}\right) \leq 1 .
$$

Remark 2.6. i) If $Z_{1}=\cdots=Z_{d}=Z$ with $Z$ a unit Fréchet random variable, then the zero-homogeneity of $\Psi_{i}^{\prime}$ 's, (2.5) and (2.8) imply that

$$
\mu(H, Q)=\sum_{i=1}^{d} \Psi_{i}(1, \ldots, 1) \mathbb{E}\left\{\frac{1}{Z}\right\}=\sum_{i=1}^{d} \Psi_{i}(1, \ldots, 1)=\mathbb{E}\left\{\max _{1 \leq i \leq d} Y_{i}\right\}=-\ln H(1, \ldots, 1) \geq 1 .
$$

Further, by (2.9) we have $\lambda(Q, H)=\mathbb{E}\left\{\min _{1 \leq i \leq d} Y_{i}\right\}$.

ii) In view of [8, Th. 2.2] (see also Eq. (6.9) in [22]), for $H$ with de Haan representation (2.6) it holds that

$$
\lambda(H, H)=-\mathbb{E}\left\{\frac{1}{\ln H\left(Y_{1}, \ldots, Y_{d}\right)}\right\},
$$

which together with (2.10) implies that

$$
\mu\left(H_{\infty}, H_{\infty}\right)=\lambda\left(H_{\infty}, H_{\infty}\right)=1,
$$

and thus the lower bound in (1.8) is sharp. We note in passing that there are numerous papers where $\lambda_{n}\left(F^{n}, F\right)$ and $\lambda(H, H)$ appear, see $[5,7,14,15,22]$ and the references therein.

iii) For common max-stable dfs $H$ the spectral random vector $\boldsymbol{Y}$ that defines (2.4) is explicitly known. Consequently, for any given random vector $\boldsymbol{Z}$, using the first expression in (2.8) and (2.9), we can easily evaluate $\mu(H, Q)$ and $\lambda(Q, H)$ by Monte Carlo simulations, respectively.

\section{Applications}

In multivariate extreme value theory it is important to have conditions that show if a given max-stable df $H$ is equal to $H_{0}$. In case $d=2$ it is well-known that $H=H_{0}$ if and only if $\lambda(H, H)=0$, see [8, Pr. 2.2] or [12, Th. 2]. Consequently, when $d>2$, in view of [9, Th. 4.3.3] we have that $H=H_{0}$ if and only if

$$
\lambda\left(H_{K}, H_{K}\right)=0,
$$

for any index set $K \subset\{1, \ldots, d\}$ with two elements. Therefore, in the sequel we consider for simplicity the case $d=2$ discussing some tractable conditions that are equivalent to $H=H_{0}$ and (1.6).

As in Balkema and Resnick [1], for a given bivariate df $H$ with unit Fréchet margins define $\xi_{H}:(0, \infty)^{2} \rightarrow$ $[0,1]$ as

$$
\xi_{H}\left(x_{1}, x_{2}\right)=\lim _{h \rightarrow 0} \frac{\left[B-H\left(\left(x_{1}, x_{2}\right)+(h,-h)\right)\right]\left[B-H\left(\left(x_{1}, x_{2}\right)+(-h, h)\right)\right]}{A\left[A+B-H\left(\left(x_{1}, x_{2}\right)+(h,-h)\right)-H\left(\left(x_{1}, x_{2}\right)+(-h, h)\right)\right]}, \quad\left(x_{1}, x_{2}\right) \in(0, \infty)^{2},
$$

where $A=H\left(x_{1}, x_{2}\right)$ and $B=H\left(x_{1}+h, x_{2}+h\right)$. If $H$ is a continuous max-id df, then in view of [1] the function $\xi_{H}$ is non-negative, measurable and bounded by 1 , almost everywhere with respect to $d H$.

Proposition 3.1. Let $H$ and $Q$ be two bivariate $d f s$ with unit Fréchet marginals. If $H$ is a max-id $d f$, then we have

$$
\lambda(Q, H)=\int_{(0, \infty)^{2}}\left[1-\xi_{H}\left(x_{1}, x_{2}\right)\right] \frac{Q\left(x_{1}, x_{2}\right)}{H\left(x_{1}, x_{2}\right)} d H\left(x_{1}, x_{2}\right) .
$$

Moreover, if $H$ is a max-stable $d f$, then the following conditions are equivalent: 
i) $H=H_{0}$;

ii) $\theta(H)=-\ln H(1,1)=2$;

iii) $\mu(H, Q)=2-\lambda(Q, H)$;

iv) $\xi_{H}$ equals 1 almost everywhere $d H$;

v) $\frac{d H^{t}}{d H}=\frac{t^{2} H^{t}}{H}$ almost everywhere $d H$ for any $t>0$.

Remark 3.2. By [12, Th. 2] we have that $\lambda(H, H)=0$ is equivalent to $H=H_{0}$ and $\lambda(H, H)=1$ is equivalent to $H=H_{\infty}$.

Moreover, statement iii) above holds for any $\mathrm{df} Q$ with continuous marginal dfs, and thus $\mu(H, Q)$ and $\lambda(Q, H)$ are both dependence measures for $H$.

We conclude this section with some equivalent conditions to (1.6).

Proposition 3.3. Let $F, G$ be two continuous bivariate $d f s$ with marginal $d f s F_{i}, G_{i}, i=1,2$ satisfying $\lim _{t \rightarrow \infty} \bar{F}_{i}(t) / \bar{G}_{i}(t)=1$. If further $F_{1}, F_{2}$ satisfy $(1.4)$ and $\left(X_{1}, X_{2}\right)$ has $d f F$, then the following are equivalent:

i) F has asymptotically independent components;

ii) $\lim _{n \rightarrow \infty} n \mathbb{P}\left\{X_{1}>n, X_{2}>n\right\}=0$;

iii) $\lim _{n \rightarrow \infty} \lambda_{n}\left(G^{n}, F\right)=0$;

iv) $\lim _{n \rightarrow \infty} \mu_{n}\left(F, G^{n}\right)=2$;

v) $\lim _{n \rightarrow \infty} n \mathbb{P}\left\{G\left(X_{1}, X_{2}\right)>1-1 / n\right\}=0$.

Remark 3.4. The equivalence of i) and ii) in Proposition 3.3 is well-known and relates to Takahashi theorem, i.e., it is enough to know that the limiting max-stable $\mathrm{df} H$ is a product $\mathrm{df}$ at one point, say $(1,1)$. See for more details in the $d$-dimensional setup [9, p. 452].

Moreover, recall that the assumption $F_{i} \in M D A(\Phi)$ means that $\lim _{n \rightarrow \infty} F_{i}^{n}\left(a_{n i} x\right)=\Phi(x), x \in \mathbb{R}$ for some norming constants $a_{n i}>0, n \in \mathbb{N}$. For notational simplicity, in this paper we assume that $a_{n i}$ 's equal $n$. If this is not the case, then we need to re-formulate statement ii) in Proposition 3.3 as $n \lim _{n \rightarrow \infty} n \mathbb{P}\left\{X_{1}>\right.$ $\left.a_{n 1}, X_{2}>a_{n 2}\right\}=0$. Note that if $F \in M D A(H)$ with $H$ a max-stable df, then

$$
\lim _{n \rightarrow \infty} n \mathbb{P}\left\{X_{1}>a_{n 1}, X_{2}>a_{n 2}\right\}=2+\ln H(1,1)=2-\theta(H)=: \lambda_{F} .
$$

In the literature, $\lambda_{F}$ is commonly referred to as the coefficient of upper tail dependence of $F$, see [9] for more details.

\section{Auxiliary Results}

Lemma 4.1. Let $\left(V_{1}, \ldots, V_{d}\right)$ be a random vector with continuous marginal $d f s H_{i}, i \leq d$. If further $G$ is a $d$ dimensional $d f$ with $G\left(x_{1}, \ldots, x_{d}\right)<1$ for any $\left(x_{1}, \ldots, x_{d}\right) \in(0, \infty)^{d}$ and the upper endpoint of $H_{i}, 1 \leq i \leq d$ equals $\infty$, then we have

$$
\lim _{n \rightarrow \infty} n \mathbb{E}\left\{G^{n-1}\left(V_{1}, \ldots, V_{d}\right)\right\}=\lim _{n \rightarrow \infty} n \mathbb{P}\left\{G\left(V_{1}, \ldots, V_{d}\right)>1-\frac{1}{n}\right\}=\kappa \in[0, \infty),
$$

if either of the limits exists. Further if

$$
G\left(x_{1}, \ldots, x_{d}\right) \leq \min _{1 \leq i \leq d} H_{i}\left(x_{i}\right), \quad\left(x_{1}, \ldots, x_{d}\right) \in(0, \infty)^{d},
$$

then $\kappa \in[0,1]$.

Proof of Lemma 4.1 The proof of (4.1) follows from [16, Lemma 2.4], see also [12, Pr. 4]. Assuming (4.2), if $H$ denotes the $\mathrm{df}$ of $\left(V_{1}, \ldots, V_{d}\right)$, then we have

$$
0 \leq n \mathbb{E}\left\{G^{n-1}\left(V_{1}, \ldots, V_{d}\right)\right\} \leq n \int_{(0, \infty)^{d}} \min _{1 \leq i \leq d} H_{i}^{n-1}\left(x_{i}\right) d H\left(x_{1}, \ldots, x_{d}\right) \leq n \int_{0}^{\infty} H_{1}^{n-1}\left(x_{1}\right) d H_{1}\left(x_{1}\right)=1,
$$


establishing the proof.

Proposition 4.2. Let $F_{n}, G_{n}, n \geq 1$ be two continuous $d f$ s on $[0, \infty)^{d}$ satisfying

$$
\lim _{n \rightarrow \infty} F_{n}^{n}\left(x_{1}, \ldots, x_{d}\right)=H\left(x_{1}, \ldots, x_{d}\right), \quad \lim _{n \rightarrow \infty} G_{n}^{n}\left(x_{1}, \ldots, x_{d}\right)=Q\left(x_{1}, \ldots, x_{d}\right), \quad\left(x_{1}, \ldots, x_{d}\right) \in[0, \infty)^{d},
$$

with $H, Q$ two max-id dfs with unit Fréchet marginal dfs $\Phi$. If for all $n$ large and some $C_{1}>0$, we have

$$
G_{n}^{n}\left(x_{1}, \ldots, x_{d}\right) \leq C_{1} \sum_{1 \leq i \leq d} F_{n i}^{n}\left(x_{i}\right), \quad\left(x_{1}, \ldots, x_{d}\right) \in(0, \infty)^{d},
$$

where $F_{n i}$ is the ith marginal df of $F_{n}$, then

$$
\lim _{n \rightarrow \infty} n \int_{[0, \infty)^{d}} G_{n}^{n}\left(x_{1}, \ldots, x_{d}\right) d F_{n}\left(x_{1}, \ldots, x_{d}\right)=\int_{(0, \infty)^{d}} Q\left(x_{1}, \ldots, x_{d}\right) d v\left(x_{1}, \ldots, x_{d}\right),
$$

where $v(\cdot)$ is the exponent measure pertaining to $H$ defined on $E:=[0, \infty]^{d} \backslash\{(0, \ldots, 0)\}$. Furthermore, if for all $n$ large and any $x_{1}, \ldots, x_{d}$ positive we have

$$
1-G_{n}\left(x_{1}, \ldots, x_{d}\right) \leq C_{2} \sum_{1 \leq i \leq d} \bar{F}_{n i}\left(x_{i}\right),
$$

then

$$
\lim _{n \rightarrow \infty} n \int_{[0, \infty)^{d}}\left[1-G_{n}\left(x_{1}, \ldots, x_{d}\right)\right] d F_{n}^{n}\left(x_{1}, \ldots, x_{d}\right)=-\int_{(0, \infty)^{d}} \ln Q\left(x_{1}, \ldots, x_{d}\right) d H\left(x_{1}, \ldots, x_{d}\right) .
$$

Proof of Proposition 4.2 For notational simplicity we consider below only the case $d=2$. From the assumptions,

$$
\lim _{n \rightarrow \infty} F_{n}^{n}\left(x_{n 1}, x_{n 2}\right)=H\left(x_{1}, x_{2}\right), \quad \lim _{n \rightarrow \infty} G_{n}^{n}\left(x_{n 1}, x_{n 2}\right)=Q\left(x_{1}, x_{2}\right)
$$

holds for every sequence $\left(x_{n 1}, x_{n 2}\right) \rightarrow\left(x_{1}, x_{2}\right) \in(0, \infty)^{2}$ as $n \rightarrow \infty$.

Let $v$ be the exponent measure of $H$ defined on $E$, see [25] for details. For any $x_{0}, y_{0}$ positive, since by our assumptions

$$
\lim _{n \rightarrow \infty} n\left[1-F_{n}\left(x_{1}, x_{2}\right)\right]=-\ln H\left(x_{1}, x_{2}\right)
$$

holds locally uniformly for $\left(x_{1}, x_{2}\right) \in(0, \infty)^{2}$, using further (4.8) and [18, Lemma 9.3] we obtain

$$
\lim _{n \rightarrow \infty} \int_{\left[x_{0}, \infty\right) \times\left[y_{0}, \infty\right)} G_{n}^{n}\left(x_{1}, x_{2}\right) d\left(n F_{n}\left(x_{1}, x_{2}\right)\right)=\int_{\left[x_{0}, \infty\right) \times\left[y_{0}, \infty\right)} Q\left(x_{1}, x_{2}\right) d v\left(x_{1}, x_{2}\right)=: I\left(x_{0}, y_{0}\right) .
$$

Moreover, by (4.4)

$$
\begin{array}{ll}
n \int_{[0, \infty)^{2}} & G_{n}^{n}\left(x_{1}, x_{2}\right) d F_{n}\left(x_{1}, x_{2}\right) \\
\leq & n C_{1}\left(\int_{\left[0, x_{0}\right]} F_{n 1}^{n-1}(x) d F_{n 1}(x)+\int_{\left[0, y_{0}\right]} F_{n 2}^{n-1}(x) d F_{n 2}(x)\right)+\int_{\left[x_{0}, \infty\right) \times\left[y_{0}, \infty\right)} G_{n}^{n}\left(x_{1}, x_{2}\right) d\left(n F_{n}\left(x_{1}, x_{2}\right)\right) \\
= & C_{1}\left(F_{n 1}^{n}\left(x_{0}\right)+F_{n 2}^{n}\left(y_{0}\right)\right)+\int_{\left[x_{0}, \infty\right) \times\left[y_{0}, \infty\right)} G_{n}^{n}\left(x_{1}, x_{2}\right) d\left(n F_{n}\left(x_{1}, x_{2}\right)\right) \\
\rightarrow & C_{1}\left(e^{-1 / x_{0}}+e^{-1 / y_{0}}\right)+\int_{\left[x_{0}, \infty\right) \times\left[y_{0}, \infty\right)} Q\left(x_{1}, x_{2}\right) d v\left(x_{1}, x_{2}\right), \quad n \rightarrow \infty
\end{array}
$$




$$
\rightarrow \int_{(0, \infty)^{2}} Q\left(x_{1}, x_{2}\right) d v\left(x_{1}, x_{2}\right), \quad x_{0} \downarrow 0, y_{0} \downarrow 0,
$$

where the equality above is a consequence of the assumption that $F_{n}, G_{n}$ have continuous marginal dfs. Hence (4.5) follows and we show next (4.7). Similarly, for $x_{0}, y_{0}$ as above, one has that

$$
\begin{aligned}
& \limsup _{n \rightarrow \infty} \int_{(0, \infty)^{2}} n\left[1-G_{n}\left(x_{1}, x_{2}\right)\right] d F_{n}^{n}\left(x_{1}, x_{2}\right) \\
& =\limsup _{n \rightarrow \infty}\left[\int_{\left(\left[x_{0}, \infty\right) \times\left[y_{0}, \infty\right)\right)^{c}} n\left[1-G_{n}\left(x_{1}, x_{2}\right)\right] d F_{n}^{n}\left(x_{1}, x_{2}\right)\right. \\
& \left.+\int_{\left[x_{0}, \infty\right) \times\left[y_{0}, \infty\right)} n\left[1-G_{n}\left(x_{1}, x_{2}\right)\right] d F_{n}^{n}\left(x_{1}, x_{2}\right)\right] \\
& \leq \quad C_{2} \limsup _{n \rightarrow \infty} \int_{\left(\left[x_{0}, \infty\right) \times\left[y_{0}, \infty\right)\right)^{c}} n\left[\bar{F}_{n 1}\left(x_{1}\right)+\bar{F}_{n 2}\left(x_{2}\right)\right] d F_{n}^{n}\left(x_{1}, x_{2}\right) \\
& +\limsup _{n \rightarrow \infty} \int_{\left[x_{0}, \infty\right) \times\left[y_{0}, \infty\right)} n\left[1-G_{n}\left(x_{1}, x_{2}\right)\right] d F^{n}\left(x_{1}, x_{2}\right) \\
& \leq \quad C_{2} \limsup _{n \rightarrow \infty}\left(F_{n 1}^{n}\left(x_{0}\right)+F_{n 2}^{n}\left(y_{0}\right)\right)\left[n \bar{F}_{n 1}\left(x_{0}\right)+n \bar{F}_{n 2}\left(y_{0}\right)\right] \\
& -\int_{\left[x_{0}, \infty\right) \times\left[y_{0}, \infty\right)} \ln Q\left(x_{1}, x_{2}\right) d H\left(x_{1}, x_{2}\right) \\
& =\quad C_{2}\left[e^{-1 / x_{0}}+e^{-1 / y_{0}}\right]\left[\frac{1}{x_{0}}+\frac{1}{y_{0}}\right]-\int_{\left[x_{0}, \infty\right) \times\left[y_{0}, \infty\right)} \ln Q\left(x_{1}, x_{2}\right) d H\left(x_{1}, x_{2}\right) \\
& \rightarrow \quad-\int_{(0, \infty)^{2}} \ln Q\left(x_{1}, x_{2}\right) d H\left(x_{1}, x_{2}\right), \quad x_{0} \downarrow 0, y_{0} \downarrow 0,
\end{aligned}
$$

hence the proof follows.

Remark 4.3. The validity of (4.4) has been shown under the assumption that $G_{n}$ is a continuous df. From the proof above it is easy to see that (4.4) still holds if we assume instead that $G_{n}$ is continuous and positive such that $G_{n}^{n}$ is a df. Similarly, for the validity of (4.7) it is enough to assume that $F_{n}^{n}$ is a continuous df.

Corollary 4.4. If $H$ is a bivariate max-stable $d f$ with unit Fréchet marginal $d f s H_{1}$ and $H_{2}$, then for $u$, $t$ positive

$$
\int_{(0, \infty)^{2}} \min \left(H_{1}^{1 / u}\left(x_{1}\right), H_{2}^{1 / t}\left(x_{2}\right)\right) d v\left(x_{1}, x_{2}\right)=u+t+\ln H(1 / u, 1 / t) .
$$

Proof of Corollary 4.4 The proof follows using Fubini Theorem and the homogeneity property of the exponent measure inherited by (1.1). We give below an alternative proof. Let $\left(V_{1}, V_{2}\right)$ have df $H$ and set $U_{i}=H_{i}\left(V_{i}\right), i=1,2$. By the assumptions since the df $H$ is continuous, applying Theorem 2.3 and (4.1) with $u, t>0$ we obtain

$$
\begin{aligned}
& \int_{(0, \infty)^{2}} \min \left(H_{1}^{1 / u}\left(x_{1}\right), H_{2}^{1 / t}\left(x_{2}\right)\right) d v\left(x_{1}, x_{2}\right) \\
& =\lim _{n \rightarrow \infty} n \int_{(0, \infty)^{2}} \min \left(H_{1}^{n / u}\left(x_{1}\right), H_{2}^{n / t}\left(x_{2}\right)\right) d H\left(x_{1}, x_{2}\right) \\
& =\lim _{n \rightarrow \infty} n \mathbb{P}\left\{\min \left(H_{1}^{1 / u}\left(V_{1}\right), H_{2}^{1 / t}\left(V_{2}\right)\right)>1-\frac{1}{n}\right\}
\end{aligned}
$$




$$
=\lim _{n \rightarrow \infty} n \mathbb{P}\left\{U_{1}>1-\frac{u}{n}, U_{2}>1-\frac{t}{n}\right\}=u+t+\ln H(1 / u, 1 / t),
$$

establishing the proof.

\section{Proofs}

Proof of Theorem 2.1 For $n>0$ set $A_{n}=Q^{1 / n}$ and $B_{n}=H^{1 / n}$. Since $H$ is a max-id df, then $B_{n}$ is a df for any $n>0$. Furthermore, since $H_{i}=Q_{i}, i \leq d$ (recall $H_{i}, Q_{i}$ are the marginal dfs of $H$ and $Q$, respectively), it can be easily checked that we can apply Proposition 4.2, which together with Remark 4.3 implies

$$
\begin{aligned}
\lim _{n \rightarrow \infty} n \int_{\mathbb{R}^{d}}\left[1-H^{1 / n}\left(x_{1}, \ldots, x_{d}\right)\right] d Q\left(x_{1}, \ldots, x_{d}\right) \\
=\lim _{n \rightarrow \infty} n \int_{\mathbb{R}^{d}}\left[1-B_{n}\left(x_{1}, \ldots, x_{d}\right)\right] d A_{n}^{n}\left(x_{1}, \ldots, x_{d}\right) \\
\left.=-\int_{\mathbb{R}^{d}} \ln H\left(x_{1}, \ldots, x_{d}\right)\right] d Q\left(x_{1}, \ldots, x_{d}\right)=\mu(H, Q) .
\end{aligned}
$$

The second claim in (2.1) follows with similar arguments and therefore we omit its proof. Next, for any non-empty subset $K$ of $\{1, \ldots, d\}$ with $m=|K|$ elements by (2.1) one has

$$
\mu\left(H_{K}, Q_{K}\right)=\lim _{n \rightarrow \infty} n \int_{\mathbb{R}^{m}}\left[1-F_{n, K}\left(x_{1}, \ldots, x_{d}\right)\right] d Q_{K}\left(x_{1}, \ldots, x_{d}\right)
$$

and

$$
\lambda\left(Q_{K}, H_{K}\right)=\lim _{n \rightarrow \infty} n \int_{\mathbb{R}^{m}} Q_{K}\left(x_{1}, \ldots, x_{d}\right) d F_{n K}\left(x_{1}, \ldots, x_{d}\right),
$$

where $F_{n K}, Q_{K}$ are the marginals of $F_{n}$ and $Q$ with respect to $K$. Note that for notational simplicity we write the marginal dfs with respect to $K$ as functions of $x_{1}, \ldots, x_{d}$ and not as functions of $x_{j 1}, \ldots, x_{j m}$ where $K=$ $\left\{j_{1}, \ldots, j_{m}\right\}$ has $m=|K|$ elements. By Fubini Theorem

$$
\int_{\mathbb{R}^{m}} Q_{K}\left(x_{1}, \ldots, x_{d}\right) d F_{n K}\left(x_{1}, \ldots, x_{d}\right)=\int_{\mathbb{R}^{m}} \bar{F}_{n K}\left(x_{1}, \ldots, x_{d}\right) d Q_{K}\left(x_{1}, \ldots, x_{d}\right),
$$

where $\bar{F}_{n K}$ stands for the joint survival function of $F_{n K}$. In the light of the inclusion-exclusion formula

$$
1-F_{n}\left(x_{1}, \ldots, x_{d}\right)=\sum_{1 \leq i \leq d}(-1)^{i+1} \sum_{K \subset\{1, \ldots, d\},|K|=i} \bar{F}_{n K}\left(x_{1}, \ldots, x_{d}\right), \quad\left(x_{1}, \ldots, x_{d}\right) \in \mathbb{R}^{d} .
$$

Using further the fact that $H$ and $Q$ have the same marginal dfs, for any index set $K$ with only one element we have

$$
\lim _{n \rightarrow \infty} n \int_{\mathbb{R}^{d}} \bar{H}_{n K}\left(x_{1}, \ldots, x_{d}\right) d Q\left(x_{1}, \ldots, x_{d}\right)=\lim _{n \rightarrow \infty} n \int_{0}^{1}\left(1-t^{1 / n}\right) d t=1,
$$

hence

$$
\begin{aligned}
\mu(H, Q) & =\lim _{n \rightarrow \infty} n \int_{\mathbb{R}^{d}}\left[1-F_{n}\left(x_{1}, \ldots, x_{d}\right)\right] d Q\left(x_{1}, \ldots, x_{d}\right) \\
& =d+\lim _{n \rightarrow \infty} n \int_{\mathbb{R}^{d}} \sum_{2 \leq i \leq d}(-1)^{i+1} \sum_{K \subset\{1, \ldots, d\},|K|=i} \bar{F}_{n K}\left(x_{1}, \ldots, x_{d}\right) d Q\left(x_{1}, \ldots, x_{d}\right) \\
& =d+\sum_{2 \leq i \leq d}(-1)^{i+1} \lim _{n \rightarrow \infty} n \int_{\mathbb{R}^{d}} \sum_{K \subset\{1, \ldots, d\},|K|=i} \bar{F}_{n K}\left(x_{1}, \ldots, x_{d}\right) d Q_{K}\left(x_{1}, \ldots, x_{d}\right)
\end{aligned}
$$




$$
=d+\sum_{2 \leq i \leq d}(-1)^{i+1} \sum_{K \subset\{1, \ldots, d\},|K|=i} \lambda\left(Q_{K}, H_{K}\right)
$$

and thus (2.2) follows. Since by the inclusion-exclusion formula we have further

$$
\bar{F}_{n}\left(x_{1}, \ldots, x_{d}\right)=\sum_{1 \leq i \leq d}(-1)^{i+1} \sum_{K \subset\{1, \ldots, d\},|K|=i}\left[1-F_{n K}\left(x_{1}, \ldots, x_{d}\right)\right], \quad\left(x_{1}, \ldots, x_{d}\right) \in \mathbb{R}^{d}
$$

the claim in (2.3) follows with similar arguments as above.

ProOF OF ThEOREM 2.5 The claim in (2.8) follows by the de Haan and inf-argmax representation of $H$. Since by the independence of $Y_{i}$ 's and $Z_{i}$ 's and the fact that $\mathbb{E}\left\{Y_{i}\right\}=\mathbb{E}\left\{1 / Z_{i}\right\}=1$ we have that

$$
\mathbb{E}\left\{Y_{i} / Z_{i}\right\}=\mathbb{E}\left\{Y_{i}\right\} \mathbb{E}\left\{1 / Z_{i}\right\}=1
$$

is valid for any $i \leq d$. Consequently, by (2.3), (2.8) and the fact that for given constants $c_{1}, \ldots, c_{d}$

$$
\min _{1 \leq i \leq d} c_{i}=\sum_{i=1}^{d}(-1)^{i+1} \sum_{K \subset\{1, \ldots, d\}:|K|=i} \max _{j \in K} c_{j},
$$

then we have

$$
\begin{aligned}
\lambda(Q, H) & =\sum_{i=1}^{d} \mathbb{E}\left\{\frac{Y_{i}}{Z_{i}}\right\}+\sum_{2 \leq i \leq d}(-1)^{i+1} \sum_{K \subset\{1, \ldots, d\},|K|=i} \mathbb{E}\left\{\max _{j \in K} \frac{Y_{j}}{Z_{j}}\right\} \\
& =\mathbb{E}\left\{\min _{1 \leq j \leq d} \frac{Y_{j}}{Z_{j}}\right\},
\end{aligned}
$$

establishing (2.9).

Further, since (5.2) holds, then by de Haan representation of max-stable dfs we have that the $\mathrm{dfs} H^{\star}, \tilde{H}$ defined in (2.6) and (2.7), respectively are max-stable with unit Fréchet marginal dfs. Hence (2.8) implies that $\mu(H, Q)=$ $\theta\left(H^{\star}\right)$. Note in passing that for $Q=H$ this follows also from [8, Pr. 2.2].

Using again that $Y_{i}$ 's are independent of $Z_{i}$ 's and $\mathbb{E}\left\{Y_{i}\right\}=1, i \leq d$ we obtain (recall $Y_{i}$ 's and $Z_{i}$ 's are nonnegative random variables)

$$
\begin{aligned}
\mu(H, Q) & =\mathbb{E}\left\{\mathbb{E}\left\{\max _{1 \leq i \leq d} \frac{Y_{i}}{Z_{i}} \mid\left(Z_{1}, \ldots, Z_{d}\right)\right\}\right\} \\
& \geq \mathbb{E}\left\{\max _{1 \leq i \leq d} \frac{\mathbb{E}\left\{Y_{i}\right\}}{Z_{i}}\right\} \\
& \geq \mathbb{E}\left\{\max _{1 \leq i \leq d} \frac{1}{Z_{i}}\right\}=\theta(\tilde{H}) \\
& \geq \max _{1 \leq i \leq d} \mathbb{E}\left\{\frac{1}{Z_{i}}\right\}=1 .
\end{aligned}
$$

With the same arguments using now that $\mathbb{E}\left\{1 / Z_{i}\right\}=1, i \leq d$ we have

$$
\begin{aligned}
\mu(H, Q) & =\mathbb{E}\left\{\mathbb{E}\left\{\max _{1 \leq i \leq d} \frac{Y_{i}}{Z_{i}} \mid\left(Y_{1}, \ldots, Y_{d}\right)\right\}\right\} \\
& \geq \mathbb{E}\left\{\max _{1 \leq i \leq d} Y_{i}\right\}=-\ln H(1, \ldots, 1)=\theta(H) .
\end{aligned}
$$

The lower bound in (2.11) follows with similar arguments, hence the proof is complete.

Proof OF Theorem 2.3 Suppose without loss of generality that $F$ satisfies (1.5). If $F_{i}=G_{i}, i=1,2$, then the claim follows from Lemma 4.1 and Proposition 4.2. We consider next the general case that $F_{i}$ 's are tail equivalent to $G_{i}$ 's and suppose for simplicity that $d=2$. In view of [16, Lemma 2.4$]$ we have

$$
\lim _{n \rightarrow \infty} n \int_{[0, \infty)} G_{i}^{n}(x) d F_{i}(x)=c_{i} \in[0, \infty), \quad i=1,2
$$


if and only if $\lim _{n \rightarrow \infty} n \mathbb{P}\left\{G_{i}\left(X_{i}\right)>1-1 / n\right\}=c_{i}$ or equivalently

$$
\lim _{x \rightarrow \infty} \frac{\bar{F}_{i}(x)}{\bar{G}_{i}(x)}=c_{i} .
$$

By the assumption $c_{i} \in(0, \infty)$ for $i=1,2$. Consequently, for all $x>0$ there exist $a_{1}, a_{2}$ positive such that

$$
a_{1} \bar{F}_{i}(x) \leq \bar{G}_{i}(x) \leq a_{2} \bar{F}_{i}(x) .
$$

Assume for simplicity that $c_{i}=1, i=1,2$. By the assumptions

$$
n \bar{F}_{i}(n x) \rightarrow 1 / x, \quad n \bar{G}_{i}(n x) \rightarrow 1 / x, \quad n \rightarrow \infty
$$

uniformly for $x$ in $[t, \infty), t>0$. Further, for $i=1,2$ we have

$$
\lim _{t \downarrow 0} \lim _{n \rightarrow \infty} n \int_{[0, t]} G_{i}^{n}(n x) d F_{i}(n x)=\lim _{t \downarrow 0} \lim _{n \rightarrow \infty} n \int_{[0, t]} \bar{G}_{i}(n x) d F_{i}^{n}(n x)=0,
$$

which implies

$$
\lim _{t \downarrow 0} \lim _{n \rightarrow \infty} n \int_{[0, t]^{2}} G^{n}(n x, n y) d F(n x, n y)=\lim _{t \downarrow 0} \lim _{n \rightarrow \infty} n \int_{[0, t]^{2}}[1-G(n x, n y)] d F^{n}(n x, n y)=0 .
$$

As in the proof of Proposition 4.2, using that $F$ and $G$ are in the MDA of $H$ and $Q$, respectively, it follows that for any integer $k$

$$
\lim _{n \rightarrow \infty} n \int_{[0, \infty)^{2}} G^{n-k}\left(x_{1}, x_{2}\right) d F\left(x_{1}, x_{2}\right)=\int_{(0, \infty)^{2}} Q\left(x_{1}, x_{2}\right) d v\left(x_{1}, x_{2}\right)=\lambda(Q, H),
$$

and further

$$
\lim _{n \rightarrow \infty} n \int_{[0, \infty)^{2}}\left[1-F\left(x_{1}, x_{2}\right)\right] d G^{n-k}\left(x_{1}, x_{2}\right)=-\int_{(0, \infty)^{2}} \ln H\left(x_{1}, x_{2}\right) d Q\left(x_{1}, x_{2}\right)=\mu(H, Q),
$$

establishing the proof.

Proof OF Proposition 3.1 In view of Theorem 2.1, since $H$ being a max-id df implies that $H^{1 / n}$ is a df for any $n \geq 1$ we have, with $F_{n}=Q^{1 / n}$, that

$$
\begin{aligned}
\int_{(0, \infty)^{2}} Q\left(x_{1}, x_{2}\right) d v\left(x_{1}, x_{2}\right) & =\lim _{n \rightarrow \infty} n \int_{(0, \infty)^{2}} Q\left(x_{1}, x_{2}\right) d H^{1 / n}\left(x_{1}, x_{2}\right) \\
& =2-\lim _{n \rightarrow \infty} n \int_{[0, \infty)^{d}}\left[1-H^{1 / n}\left(x_{1}, x_{2}\right)\right] d F_{n}^{n}\left(x_{1}, x_{2}\right) \\
& =\int_{(0, \infty)^{2}}\left[2+\ln H\left(x_{1}, x_{2}\right)\right] d Q\left(x_{1}, x_{2}\right) .
\end{aligned}
$$

Since further by $\left[1\right.$, Th. 7] the restriction of $v$ on $(0, \infty)^{2}$ denoted by $v_{0}$ satisfies

$$
\frac{d v_{0}}{d H}=\frac{1-\xi_{H}}{H}
$$

and $\xi_{H}\left(x_{1}, x_{2}\right) \in[0,1]$ almost everywhere $d H$, then the first claim follows.

The equivalence of i) and ii) is known as Takahashi Theorem, see [9, Th. 4.3.2]. Since $\xi_{H} \in[0,1]$ almost everywhere $d H$, then the equivalence of ii) and iii) is a direct consequence of (3.3) and the fact that $\lambda(Q, H)=$ $2-\mu(H, Q)$, see (2.3). Clearly, by (3.3) we have thus $\xi_{H}=1$ almost everywhere $d H$ is equivalent to $H=H_{0}$, whereas iv) is equivalent to $\mathrm{v})$ is consequence of $[1, \mathrm{Th} .7]$. 
Proof of Proposition 3.3 If $F$ (1.6) holds, then clearly ii) is satisfied and thus i) implies ii). If ii) holds, then

$$
\begin{aligned}
\limsup _{n \rightarrow \infty} F^{n}\left(n x_{1}, n x_{2}\right) & =\exp \left(\limsup _{n \rightarrow \infty} n \ln \left(1-\left[1-F\left(n x_{1}, n x_{2}\right)\right]\right)\right) \\
& =\exp \left(-\limsup _{n \rightarrow \infty} n\left(1-F\left(n x_{1}, n x_{2}\right)\right)\right) \\
& \leq \exp \left(-\limsup _{n \rightarrow \infty}\left[n \bar{F}_{1}\left(n x_{1}\right)+n \bar{F}_{2}\left(n x_{2}\right)-n \mathbb{P}\left\{X_{1}>n \min \left(x_{1}, x_{2}\right), X_{2}>n \min \left(x_{1}, x_{2}\right)\right\}\right]\right) \\
& =\exp \left(-1 / x_{1}-1 / x_{2}\right), \quad x_{1}, x_{2}>0 .
\end{aligned}
$$

As for the derivation of (1.2) we obtain further

$$
\liminf _{n \rightarrow \infty} F^{n}\left(n x_{1}, n x_{2}\right) \geq \exp \left(-1 / x_{1}-1 / x_{2}\right), \quad x_{1}, x_{2}>0
$$

implying that $F \in M D A\left(H_{0}\right)$, hence i) follows.

Assuming iii) and since the marginal dfs of $G$ are in the MDA of $\Phi$, with the same calculations as in (5.3) for the $\operatorname{df} G$ we obtain

$$
\begin{aligned}
0=\lim _{n \rightarrow \infty} n \int_{[0, \infty)^{2}} G^{n}\left(x_{1}, x_{2}\right) d F\left(x_{1}, x_{2}\right) & \geq \lim _{n \rightarrow \infty} n \mathbb{P}\left\{X_{1}>n, X_{2}>n\right\} G^{n}(n, n) \\
& \geq c \lim _{n \rightarrow \infty} n \mathbb{P}\left\{X_{1}>n, X_{2}>n\right\},
\end{aligned}
$$

for some $c \in\left(0, e^{-2}\right)$, hence ii) follows.

Next, assume that ii) holds. We have that $G\left(x_{1}, x_{2}\right) \leq G_{1}\left(x_{1}\right) G_{2}\left(x_{2}\right)=: K\left(x_{1}, x_{2}\right)$ and by the assumption that $G_{i}$ 's are in the MDA of $\Phi$ it follows that $K$ is in the MDA of $H_{\infty}$. Further ii) implies that $F \in M D A\left(H_{0}\right)$ and $-\ln H(1,1)=2$. Consequently, Theorem 2.3 yields

$$
\lim _{n \rightarrow \infty} \lambda_{n}\left(K^{n}, F\right)=\lambda\left(H_{\infty}, H_{0}\right)
$$

But from Corollary 4.4 we have that $\lambda\left(H_{\infty}, H_{0}\right)=0$, hence ii) implies iii).

Let $\bar{G}$ be the joint survival function of the bivariate $\mathrm{df} G$. For any positive integer $n$, we have that $F^{n}$ is a bivariate df. Hence by Fubini theorem and the fact that $F_{i}=G_{i}, i=1,2$ are continuous dfs, for any positive integer $n$ we obtain

$$
\begin{aligned}
\int_{\mathbb{R}^{2}} F^{n}\left(x_{1}, x_{d}\right) d G\left(x_{1}, x_{2}\right) & =\int_{\mathbb{R}^{2}} \bar{G}\left(x_{1}, x_{2}\right) d F^{n}\left(x_{1}, x_{2}\right) \\
& =2 n /(n+1)-\int_{\mathbb{R}^{2}}\left[1-G\left(x_{1}, x_{2}\right)\right] d F^{n}\left(x_{1}, x_{2}\right)
\end{aligned}
$$

and thus the equivalence of iii) and iv) follows. The equivalence iv) and v) follows from Lemma 4.1 and thus the proof is complete.

Acknowledgement: I am in debt to both reviewers for numerous suggestions and corrections. Support from SNSF Grant 200021-175752/1 is kindly acknowledged.

\section{References}

[1] Balkema, A. A. and S. I. Resnick (1977). Max-infinite divisibility. J. Appl. Probability 14(2), 309-319.

[2] Beirlant, J., Y. Goegebeur, J. Teugels, and J. Segers (2004). Statistics of Extremes. John Wiley \& Sons, Chichester.

[3] Dębicki, K. and E. Hashorva (2017). Approximation of supremum of max-stable stationary processes and Pickands constants. Available at https://arxiv.org/abs/1712.04243.

[4] de Haan, L. (1984). A spectral representation for max-stable processes. Ann. Probab. 12(4), 1194-1204. 
[5] de Haan, L. (1985). Extremes in higher dimensions: The model and some statistics. In Proceedings of the 45th session of the International Statistical Institute. International Statistical Institute, Voorburg.

[6] Dȩbicki, K. and M. Mandjes (2015). Queues and Lévy Fluctuation Theory. Springer, Cham.

[7] Dombry, C., M. Falk, and M. Zott (2018). On functional records and champions. J. Theoret. Probab., to appear. Available at https://doi.org/10.1007/s10959-018-0811-7.

[8] Dombry, C., M. Ribatet, and S. Stoev (2017). Probabilities of concurrent extremes. J. Amer. Statist. Assoc., to appear. Available at https://doi.org/10.1080/01621459.2017.1356318.

[9] Falk, M., J. Hüsler, and R.-D. Reiss (2011). Laws of Small Numbers: Extremes and Rare Events. Third edition. Birkhäuser, Basel.

[10] Gnedin, A. V. (1993). On multivariate extremal processes. J. Multivariate Anal. 46(2), 207-213.

[11] Gnedin, A. V. (1994a). Conical extremes of a multivariate sample. J. Res. Natl. Inst. Stand. Technol. 99(4), 511-519.

[12] Gnedin, A. V. (1994b). On a best-choice problem with dependent criteria. J. Appl. Probab. 31(1), 221-234.

[13] Gnedin, A. V. (1998). Records from a multivariate normal sample. Statist. Probab. Lett. 39(1), 11-15.

[14] Gnedin, A. V. (2008). Corners and records of the Poisson process in quadrant. Electron. Commun. Probab. 13, 187-193.

[15] Gnedin, A. V. and A. Marynych (2012). Exponential-uniform identities related to records. Electron. Commun. Probab. 17, no. 26, $5 \mathrm{pp}$.

[16] Hashorva, E. (2002a). Asymptotics of dominated Gaussian maxima. Extremes 5(4), 353-368.

[17] Hashorva, E. (2002b). Remarks on domination of maxima. Statist. Probab. Lett. 60(1), 101-109.

[18] Hashorva, E. (2017). Representations of max-stable processes via exponential tilting. Stochastic Process. Appl., to appear. Available at https://doi.org/10.1016/j.spa.2017.10.003.

[19] Hashorva, E. and J. Hüsler (2001). On the number of points near the multivariate maxima. Statist. Probab. Lett. 55(2), 113124.

[20] Hashorva, E. and J. Hüsler (2002). On asymptotics of multivariate integrals with applications to records. Stoch. Models 18(1), 41-69.

[21] Hashorva, E. and J. Hüsler (2005). Multiple maxima in multivariate samples. Statist. Probab. Lett. 75(1), 11-17.

[22] Ledford, A. W. and J. A. Tawn (1998). Concomitant tail behaviour for extremes. Adv. in Appl. Probab. 30(1), $197-215$.

[23] Molchanov, I. and K. Strokorb (2016). Max-stable random sup-measures with comonotonic tail dependence. Stochastic Process. Appl. 126(9), 2835-2859.

[24] Planinić, H. and P. Soulier (2018). The tail process revisited. Extremes, to appear. Available at https://doi.org/10.1007/ s10687-018-0312-1.

[25] Resnick, S. I. (1987). Extreme Values, Regular Variation and Point Processes. Springer, New York. 